\title{
Note sur l'évolution de la composition chimique du fœtus et du nouveau-né ovin de race Ile-de-France
}

\author{
Yseult VILLETTE (*) et M. THERIEZ \\ avec la collaboration technique de J. LERoux \\ I.N.R.A., Laboratoire de la Production ovine \\ Centre de Recherches de Clermont-Ferrand \\ Theix, F 63122 Ceyrat
}

\begin{abstract}
Résumé
Nous avons déterminé sur 15 fotus Ile-de-France prélevés par césarienne entre 99 et 144 jours de gestation et sur 16 agneaux nouveau-nés de même race choisis de poids variable à la naissance les teneurs en eau, cendres, azote et énergie. Pendant la gestation la teneur en eau diminue tandis que celles en cendres, azote et énergie augmentent. En fin de gestation l'accrétion lipidique semble cependant ralentir. La composition chimique de l'agneau nouveau-né a été indépendante du poids à la naissance.
\end{abstract}

Mots clés : Agneau(x), nouveau-né(s), fatus, composition chimique.

\section{Introduction}

Le calcul des besoins de la brebis gestante nécessite de connaître l'évolution des quantités de nutriments (énergie, protéines, minéraux...) fixées par le fótus et ses annexes au cours de la gestation. Ces quantités peuvent être estimées à partir du poids de la portée à la naissance et de la composition chimique du nouveau-né (I.N.R.A., 1978). Les équations d'estimation proposées par l'I.N.R.A. ont été effectuées à partir d'une compilation de données sur la composition chimique de fœus de races étrangères. Nous avons publié plus récemment des données de composition chimique d'agneaux nouveau-nés de différentes races (Villette \& Aurousseau, 1981) et, contrairement à d'autres auteurs, il n'était pas apparu d'effet propre du poids à la naissance sur sa composition rapportée au $\mathrm{kg}$ de poids vif à la naissance. Nous présentons ici des résultats complémentaires obtenus sur 15 fœtus Ile-de-France, race à fort potentiel de croissance prélevés par césarienne à $99,119,132$ et 144 jours de gestation et sur 16 agneaux nouveau-nés de même race issus de portées de 1 à

(*) Adresse actuelle : Laboratoire de Recherches de la Chaire de Zootechnie, I.N.R.A. I.N.A.P.G., 16, rue Claude-Bernard, 75231 Paris cedex 05. 


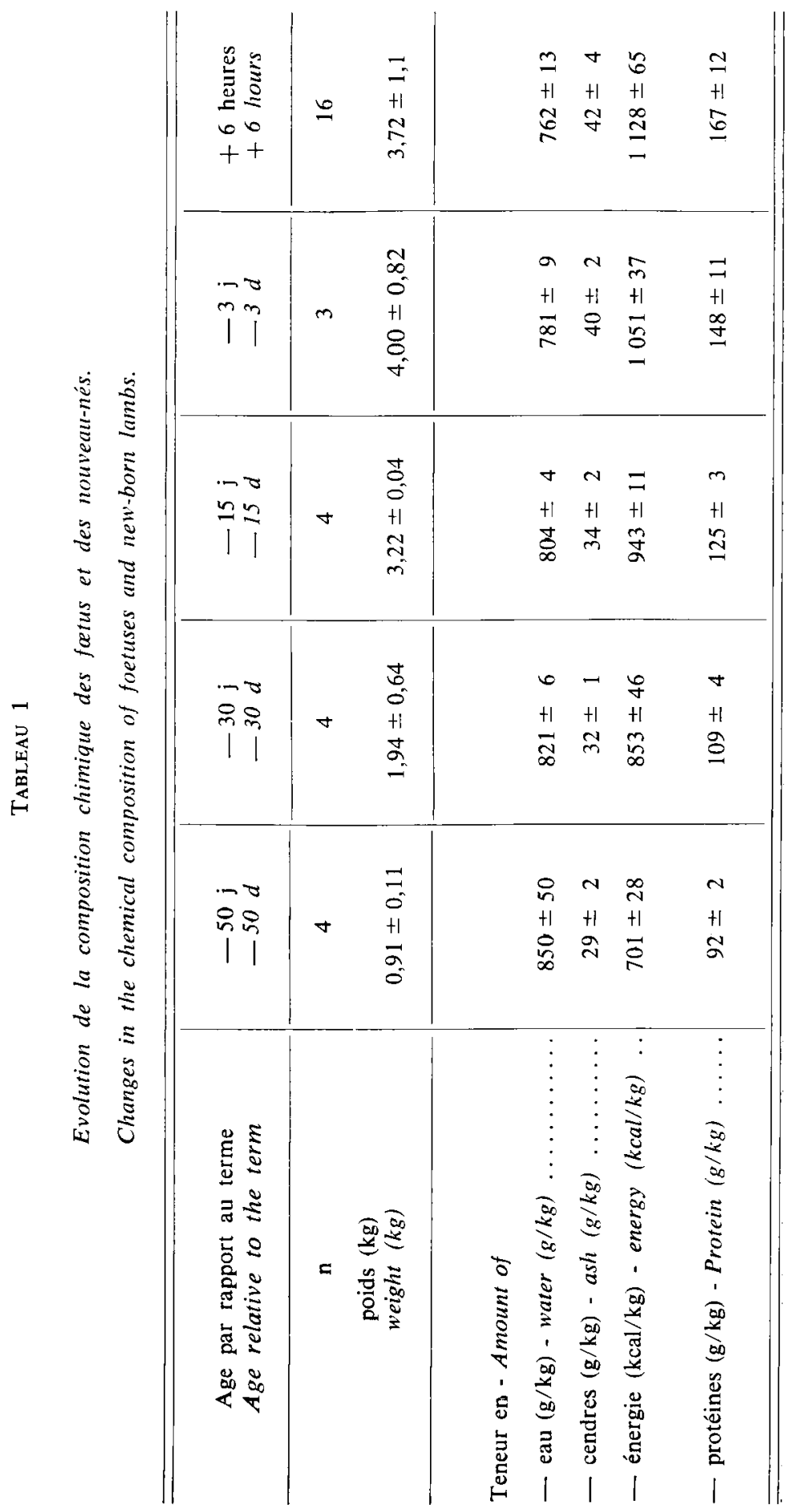


3 agneaux ( 3 simples, 10 doubles et 3 triples) et pesant de 2,4 à 6,8 $\mathrm{kg}$. Ces agneaux ont été abattus 6 heures après leur naissance sans avoir jamais tété. La conservation et le traitement des échantillons sont similaires à ceux présentés dans la publication précédente (Villette \& Aurousseau, 1981).

\section{Evolution de la composition chimique du fotus et du nouveau-né}

Pendant les 50 derniers jours de gestation, la composition chimique du fotus évolue fortement : sa teneur en eau diminue tandis que celles en cendres, énergie et matières azotées $(\mathrm{N} \times 6,25)$ augmentent (tabl. 1). Ces valeurs et leur évolution sont similaires à celles obtenues par d'autres auteurs et ayant pour certaines servi de base à la détermination des équations proposées par l'I.N.R.A. (1978). Seules les valeurs obtenues par RatTRay et al. (1974) s'en éloignent. Rapportées à la matière sèche, les teneurs en cendres restent stables pendant la période étudiée (17 à 19 p. 100), celles en protéines croissent légèrement de 61 p. 100 à 70 p. 100 . La valeur énergétique de la matière sèche augmente entre -50 jours $(4670 \mathrm{kcal} / \mathrm{kg})$ et -15 jours $(4810 \mathrm{kcal} / \mathrm{kg}$ ), puis se stabilise. Ceci suggère vraisemblablement un ralentissement de l'accrétion lipidique en fin de gestation, résultat comparable à ceux de Mac DonalD et al. (1979). L'ensemble de ces résultats sur l'évolution de la composition chimique des fœtus ovins correspond donc aux données disponibles sur l'évolution de la composition corporelle : celle-ci se caractérise en fin de gestation par un développement du squelette et surtout du tissu musculaire tandis que la croissance du tissu adipeux reste isométrique (Robelin, Villette \& Etienne, 1984).

\section{Tableau 2}

Estimation des quantités journalières

fixées par un foetus pesant $4 \mathrm{~kg}$ à la naissance.

Estimation of the daily accretion in a foetus weighing $4 \mathrm{~kg}$ at birth.

Age fotal par rapport au terme à

147 jours - Foetal age relative to

birth at 147 days.............................

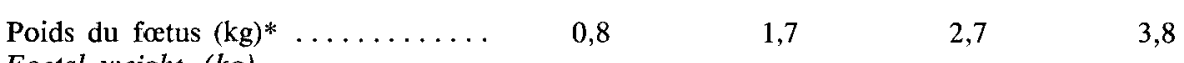

Foetal weight $(\mathrm{kg})$

Quantités fixées par le fœus en :

Foetal accretion of

- cendres $(\mathrm{g} / \mathrm{j})$ - ash $(g / d) \ldots \ldots$

- énergie $(\mathrm{kcal} / \mathrm{j})$ - energy $(\mathrm{kcal} / \mathrm{d})$

- protéines $(\mathrm{g} / \mathrm{j})$ - Proteins $(g / d)$

$\begin{array}{cccc}-50 & -30 & -15 & -3 \\ 0,8 & 1,7 & 2,7 & 3,8\end{array}$

* Poids fœtal (P) estimé à partir de l'équation I.N.R.A. (1978), P = poids à la naissance $\times \exp 2065$ $[1-\exp (-0,0103 \mathrm{t})]$.

Foetal weight (W) estimated from the I.N.R.A. (1978) equation, $W=$ birthweight $\times$ exp 2,605 $[1-\exp (-0.0103 t)]$. 
A la naissance, qui se situe vers 147 jours de gestation dans cette race (VILLETTE-Houssin \& Brelurut, 1980), la teneur en eau diminue de $781 \mathrm{~g} / \mathrm{kg}$ pour le fœtus de 144 jours à $762 \mathrm{~g} / \mathrm{kg}$ pour l'agneau nouveau-né (tabl. 1). La présence de liquide amniotique imbibant la toison doit être le facteur principal de cette diminution de la teneur en eau.

Ces données sur la composition chimique nous ont permis d'estimer l'évolution des quantités journalières fixées par le fœtus (tabl. 2). Les valeurs obtenues sont voisines de celles proposées par l'I.N.R.A. (1978) et confirment donc les possibilités d'extension de ces données à des races à fort potentiel de croissance.

\section{TABLEAU 3}

Equations d'allométrie reliant les quantités totales $(Y)$

en eau, énergie, protéines et cendres au poids du nouveau-né $(P)$.

Allometry equations between the total quantities ( $Y)$ of water, energy, crude protein, ash, and lamb birth weight $(P)$.

\begin{tabular}{|c|c|c|c|c|c|}
\hline & $\log Y=a+b \log$ & $\sigma b^{*}$ & $\sigma e^{*}$ & $\begin{array}{l}\text { CVR } \\
(\%)^{*}\end{array}$ & $\mathrm{R}^{*}$ \\
\hline \multicolumn{6}{|c|}{$\begin{array}{l}16 \text { agneaux abattus à } 6 \text { heures } \\
16 \text { lambs killed } 6 \text { hours after birth }\end{array}$} \\
\hline $\begin{array}{l}\text { Eau }(g) \ldots \ldots \cdots \\
\text { Water }(g)\end{array}$ & $2,882+1,002 \log \mathbf{P}$ & 0,013 & 0,007 & 3,6 & 0.999 \\
\hline $\begin{array}{l}\text { Cendres (g) } \ldots \ldots \\
A \operatorname{sh}(g)\end{array}$ & $1,641+0,956 \log \mathbf{P}$ & 0,078 & 0,041 & 8,1 & 0.959 \\
\hline $\begin{array}{l}\text { Energie }(\mathrm{kcal}) \\
\text { Energy }(\mathrm{kcal})\end{array}$ & $3,045+1,005 \log P$ & 0,041 & 0,021 & 2,1 & 0.989 \\
\hline $\begin{array}{l}\text { Protéines }(\mathrm{g}) \ldots \ldots \\
\text { Crude protein }(\mathrm{g})\end{array}$ & $2,209+1,017 \log \mathbf{P}$ & 0,054 & 0,028 & 3,8 & 0,982 \\
\hline
\end{tabular}

6 agneaux abattus à 6 heures

6 lambs killed 6 hours after birth

+12 agreaux morts à la naissance (Villette \& Aurousseau, 1981)

+12 lambs dead at birth

\begin{tabular}{|c|c|c|c|c|c|}
\hline $\begin{array}{l}\text { Eau }(g) \ldots \\
\text { Water }(g)\end{array}$ & $2,882+0,999 \log \mathrm{P}$ & 0,010 & 0,070 & 2,3 & 0,999 \\
\hline $\begin{array}{l}\text { Cendres (g) } \ldots . . \\
\text { Ash (g) }\end{array}$ & $1,642+0,934 \log \mathrm{P}$ & 0,060 & 0,039 & 9,2 & 0,958 \\
\hline $\begin{array}{l}\text { Energie (kcal) } \ldots \\
\text { Energy }(\text { kcal })\end{array}$ & $3,055+1,008 \log \mathrm{P}$ & 0,043 & 0,028 & 4,4 & 0,978 \\
\hline $\begin{array}{l}\text { Protéines }(\mathrm{g}) \ldots . . \\
\text { Crude protein }(\mathrm{g})\end{array}$ & $2,193+1,051 \log P$ & 0,045 & 0,030 & 4,4 & 0,978 \\
\hline \multicolumn{6}{|c|}{$\begin{array}{l}\text { * } \mathrm{\sigma b}: \text { Ecart type du coefficient d'allométrie. } \\
\text { Standard deviation of the coefficient of allometry }(b) \text {. }\end{array}$} \\
\hline \multicolumn{6}{|c|}{$\begin{aligned} \text { CVR : Coefficient de variation résiduel. } \\
\text { Residual coefficient of variation. }\end{aligned}$} \\
\hline \multicolumn{6}{|c|}{ 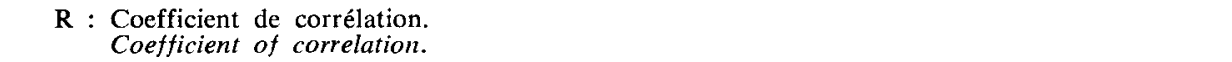 } \\
\hline
\end{tabular}




\section{Influence du poids à la naissance et du sexe sur la composition chimique de l'agneau nouveau-né}

Les quantités totales (Y) d'eau, de cendres, d'énergie et de protéines ont été reliées au poids à la naissance $(\mathrm{P})$ du nouveau-né selon les équations d'allométrie $\log \mathrm{Y}=\mathrm{a}+\mathrm{b} \log \mathbf{P}$ (tabl. 3). Les calculs ont été effectués d'une part sur 16 agneaux abattus 6 heures après la naissance et, d'autre part, sur l'ensemble des 28 données disponibles (16 + 12 agneaux morts à la naissance, Villette \& Aurousseau, 1981). Les coefficients obtenus sont voisins pour les deux séries de données. Aucun des coefficients d'allométrie n'est significativement différent de 1 et les teneurs $(\mathrm{Y} / \mathrm{kg})$ sont donc indépendantes du poids à la naissance, confirmant ainsi nos résultats antérieurs (Villette \& Aurousseau, 1981). Les divergences entre auteurs concernant l'influence du poids à la naissance sur la composition chimique de l'agneau doivent correspondre à des causes différentes de la variabilité du poids à la naissance. Quand l'alimentation maternelle est satisfaisante, il n'y aurait pas d'effet du poids à la naissance sur la composition chimique du nouveau-né. En revanche, une sous-alimentation maternelle peut se traduire non seulement par une diminution du poids mais aussi par une diminution de la teneur en lipides de l'organisme (AlEXANDER, 1962).

Les 16 agneaux abattus à la naissance comprenaient 8 mâles et 8 femelles. L'influence du sexe, testée par analyse de covariance prenant en compte la variable poids à la naissance est faible et non significative, résultat similaire à celui reporté précédemment (Villette \& Aurousseau, 1981).

\section{Summary}

Changes in the chemical composition of foetuses and new-born lambs of the Ile-de-France breed

The water, ash, crude protein and energy contents were determined in 15 Ile-de-France foetuses obtained by caesarean operation between 99 and 144 days of pregnancy and in 16 new-born lambs of the same breed with variable birthweight. During pregnancy, the water content decreased as the ash, nitrogen and energy contents increased. At the end of pregnancy, the lipid accretion seemed to slow down. The chemical composition of the new-born lambs was independent of their birthweight.

Key words : lamb(s) newborn, fetus fetus(es), chemical composition.

Reçu en juillet 1983.

Accepté en août 1983.

\section{Références bibliographiques}

Alexander G., 1962. Energy metabolism in the starved new-born lamb. Aust. J. Agric. Res., 13, 144-164.

I.N.R.A., 1978. In Alimentation des Ruminants. Reproduction, gestation, lactation, 229-243, ed. I.N.R.A. Publications, Versailles. 
Mac Donald I., Robinson J.J., Fraser C., Smart R.L., 1979. Studies on reproduction in prolific ewes. 5. Accretion of nutrient in the fetuses and adnexa. J. Agric. Sci. Camb., 92, 591-603.

Rattray P.V., Garrett W.N., East N.E., Hinman N., 1974. Growth, development and composition of the ovine conceptus and mammary gland during pregnancy. J. Anim. Sci., 38, 613-626.

Robelin J., Villette Y., Etienne M., 1984. Croissance et développement corporel. In Physiologie et pathologie périnatales, $14^{\circ}$ Journées du Grenier de Theix, 15-17 décembre 1982 (en cours de publication).

Villette-Houssin Y., Brelurut A., 1980. Variations et implications de la durée de gestation dans un troupeau ovin. Bull. Techn. C.R.Z.V. Theix-I.N.R.A., 41, 49-55.

Villette Y., Aurousseau B., 1981. Influence du poids à la naissance et du génotype sur la composition chimique de l'agneau nouveau-né. Ann. Zootech., 30, 285-298.

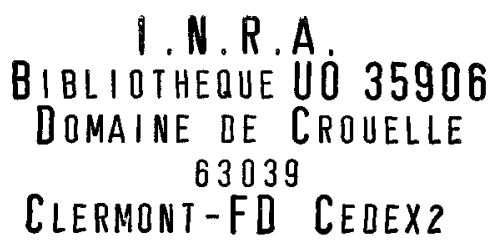

